\title{
Large-scale EM \& Correlative Microscopy (Nanotomy \& CLEM)
}

\author{
Ben N. G. Giepmans, Pascal de Boer, Klaas A. Sjollema, Jeroen Kuipers \& Anouk G.H. Wolters
}

Dept. Cell Biology, University Medical Center Groningen, Groningen, The Netherlands

A spectrum of dyes and probes now enable to localize molecules of interest within living cells by fluorescence microscopy. With electron microscopy (EM), cellular ultrastructure has been revealed. Bridging these two modalities, correlated light microscopy and EM (CLEM) opens new avenues [1].

The first focus will be on recent developed labeling strategies for molecules that allow CLEM (Fig.1). These include particles and substrates to highlight endogenous proteins that are targeted using affinity, but also genetically-encoded probes [2], and traditional stains for light microscopy that aid in EManalysis of samples. Probes that can only be detected in a single modality, and require image overlay, as well as combinatorial probes that can be visualized both at LM and EM levels will be discussed.

In addition, new approaches for large scale EM ("nanotomy"), either TEM-based [3] or S(T)EM-based $[4,5]$, to visualize macromolecules and organelles in the context of organized cell systems and tissues will be highlighted. Matching the areas of acquisition in CLEM and EM will not only increase understanding of the molecules in the context, but also is a straight forward manner to combine the LM and EM image data. While these new developments aid to better understand the contribution of molecules, organelles and cells in tissue-function, the amount of data is huge and quantification and sharing data need new solutions. We will show how millions of particles can be recognized and quantified using Fiji. Moreover, our open-access data-sharing (Fig.2) via nanotomy.org will be highlighted to easily access the ultrastructure in a variety of research projects, including blistering diseases that affect skin and mucosa in men; Type 1 diabetes research in a rat model; Flies, C. Elegans and zebrafish to study the basics of transcription regulation but also as a model of neurodegeneration.

Covering a variety of probes and approaches for image overlay will help to enable (new) users to implement CLEM to better understand how molecules (mal)function in biology [6].

\section{References:}

[1] De Boer et al. (2015) CLEM: Ultrastructure lights up! Nature Methods 12:503

[2] Kuipers et al. (2015) FLIPPER for CLEM. Cell \& Tissue Research 360:61

[3] Ravelli et al. (2013) Destruction (...) in T1D rats at macromolecular resolution. Sci. Reports 3:1804

[4] Sokol et al. (2015) Nanotomy of blistering diseases. J. Investigative Dermatology 135:1763

[5] Kuipers et al. (2015) SEM-based immunolabelling and nanotomy. Exp. Cell Res. 337:202

[6] Sponsored by ZonMW91111.006; NWO175-010-2009-023 ; STW Microscopy Valley 12718 

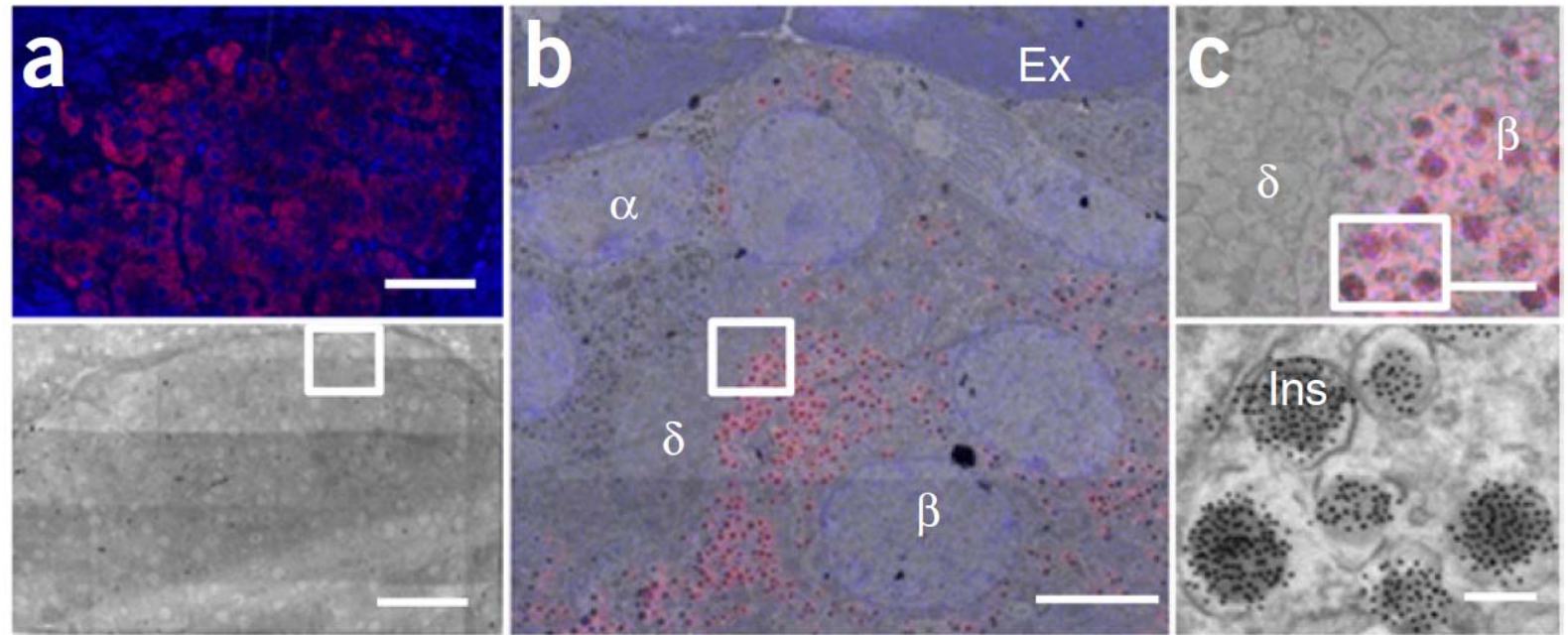

Figure 1. Examples of CLEM with distinct approaches. Post-embedding insulin immunolabeling (biotin-labeled antibodies) by QDs (streptavidin-conjugated QD655, red) and Hoechst counterstain (blue) of rat pancreas74. FM (top) followed by scanning EM (bottom) and overlay (b). (c) Boxed area from $b$ (top) and magnification (bottom). Note the detection of the electron-dense nanoparticles. Ex, exocrine pancreas; $\alpha$, alpha cell; $\beta$, beta cell; $\delta$, delta cell; Ins, insulin granule. Scale bars: $50 \mu \mathrm{m}(\mathrm{a}), 5$ $\mu \mathrm{m}$ (b), $1 \mu \mathrm{m}$ (c, top) and $200 \mathrm{~nm}$ (c, bottom). Reproduced from [1].

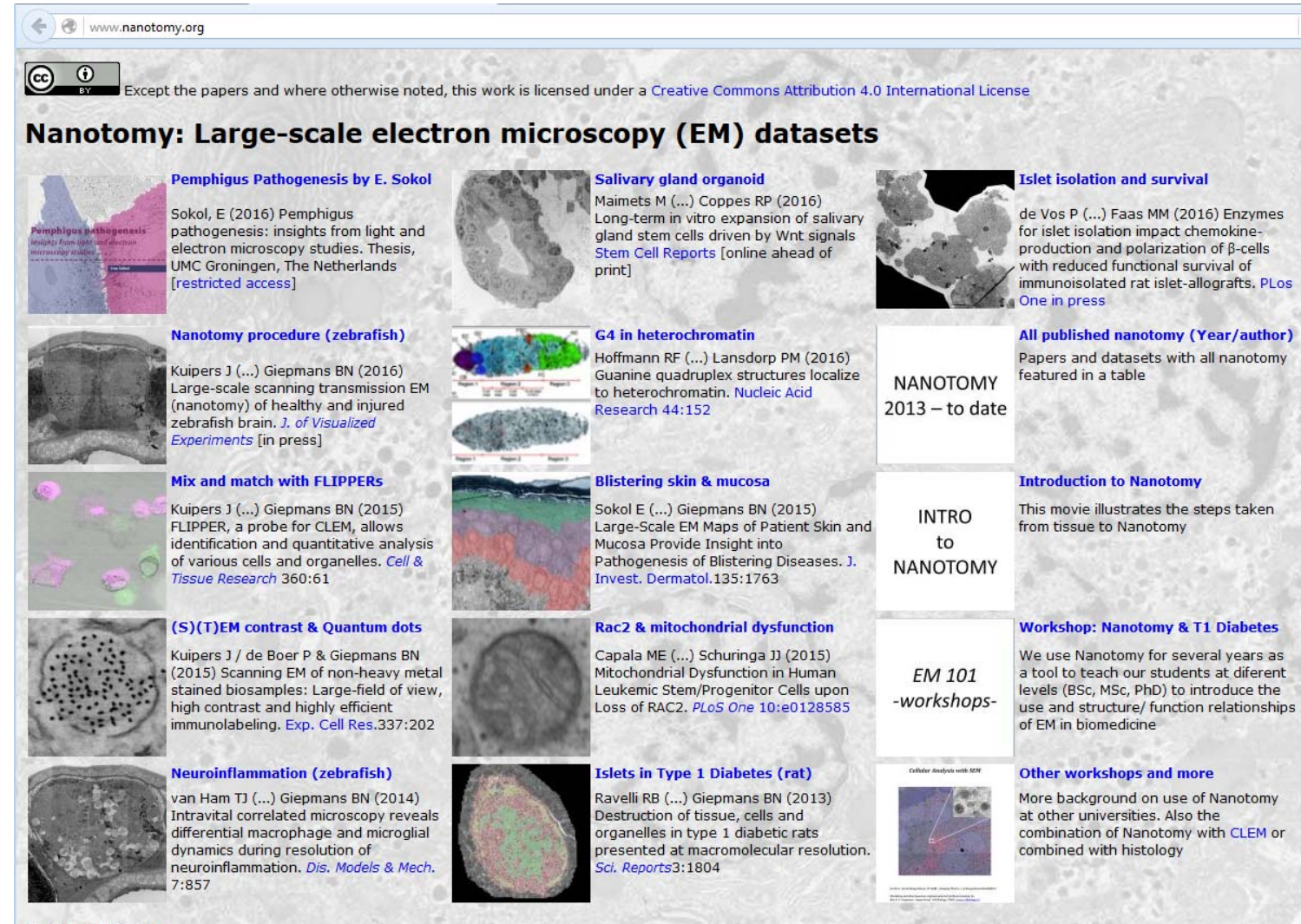

Disclaimer - Contact

Figure 2. www.nanotomy.org. The site allows access to metadata via links to publications as well as to the raw EM data in a "Google-earth"-like fashion. Over 180Gb of EM-data is now open access. 